
\title{
28 Research Square \\ Optimal treatment and complications for the perforated upper gastrointestinal tract
}

Takahito Sugase ( $\sim$ getssugase0130@gmail.com )

Kinan Hospital https://orcid.org/0000-0003-4645-2056

\section{Toshiya Michiura}

Kinan Hospital

Shoichiro Urabe

Kinan Hospital

Kazuki Sasaki

Kinan Hospital

Tsuyoshi Hata

Kinan Hospital

Nobuyasu Hayashi

Kinan Hospital

Kazuo Yamabe

Kinan Hospital

\section{Research article}

Keywords: perforated upper gastrointestinal tract, peptic ulcer, gastric cancer, conservative treatment, postoperative complications

Posted Date: August 6th, 2020

DOI: https://doi.org/10.21203/rs.3.rs-48835/v1

License: (c) (1) This work is licensed under a Creative Commons Attribution 4.0 International License.

Read Full License 


\section{Abstract}

Background The perforation of upper gastrointestinal tract, primarily caused by peptic ulcer or cancer, is afflicted by a notoriously high mortality rate. The selection of appropriate risk assessments and therapeutic alternatives becomes important when addressing the risk for morbidity and mortality. We aimed to evaluate the optimal treatment and the post-treatment complications for this condition.

Methods We retrospectively analyzed 50 patients with intraperitoneal free air due to perforated stomach or duodenum who were consecutively treated at a single institution between 2010 and 2019.

Results All patients received initial inpatient treatment that was categorized as either surgery $(n=43$, $86 \%)$ or non-surgery $(n=7,14 \%)$. The non-surgically cured patients were significantly younger and had no or localized peritonitis, no ascites, lower C-reactive protein (CRP) levels, and shorter hospital stay than the surgery patients. Of seven non-surgery patients, two patients were converted to surgery for worsening symptoms. One of them, who was elderly and had a longer perforation-to-treatment time, stayed at the hospital more than 2 months after surgery with CD Grade $\bigotimes$. Evaluation of postoperative complications using the Clavien-Dindo classification showed that the patients with Grade $\nabla-\nabla(n=21)$ were significantly older and had higher heart rates, poorer physical status, and longer perforation-to-surgery than those with Grade $0-\otimes(n=24)$. Preoperative CRP, prothrombin time, and lactate were significantly higher, and

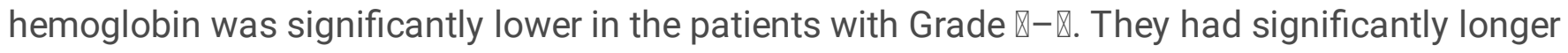
operation times and found acute renal failure more frequently. Postoperative findings showed a significantly more prolonged period of antibiotic administration, fasting, and hospital stay. The postoperative blood examinations of them showed that minor changes were observed in WBC and neutrophil, and neutrophil and CRP were significantly higher after surgery. The multivariable analyses identified elevated lactate as an independent risk factor for postoperative complications. The postoperative outcomes in patients with perforated gastric cancer depended on the stage and whether a curative resection could be performed.

Conclusions Consideration should be given to the indications of non-surgery in elderly patients as well as the delay of treatment and postoperative outcomes of patients with elevated lactate preoperatively.

\section{Background}

The perforated upper gastrointestinal tract (PUGT), stomach, or duodenum, which are mainly caused by peptic ulcer (PU) or cancer, are afflicted by a notoriously high mortality rate[1-4]. Most patients present with an acute abdominal condition with localized or generalized peritonitis, and the risk of death is increased if sepsis develops. Early diagnosis is essential, but clinical signs can be obscured in elderly patients who have a higher risk of mortality [5-8] and imaging examinations, such as chest/abdominal X-ray and computed tomography (CT) scan, play an important role in diagnosis $[5,6,9,10]$. The selection of appropriate risk assessments and therapeutic alternatives becomes important when addressing the risk for morbidity and mortality. 
The World Society of Emergency Surgery (WSES) guidelines present evidence-based international consensus statements on the management of complicated PU from a collaboration of a panel of experts. These guidelines are intended to improve the knowledge and awareness of physicians around the world on this specific topic. The aforementioned panel of experts was assembled and charged by the board of the WSES with the purpose of answering clinical questions (CQs) pertaining to six main topics that thoroughly explain this pathology. These topics are diagnosis, resuscitation, nonoperative management, surgery, angiography-angioembolization, antimicrobial therapy [11]. In Japan, the Japanese Society of Gastroenterology (JSGE) has developed the evidence-based clinical practice guidelines for PU. The JSGE guidelines consist of seven items: bleeding gastric and duodenal ulcers, Helicobacter pylori (H. pylori) eradication therapy, non-eradication therapy, drug-induced ulcer, non-H. pylori, non-nonsteroidal antiinflammatory drugs (NSAIDs) ulcer, surgical treatment, and conservative therapy for perforation and stenosis [12]. Both guidelines have provided evidence-based answers to any CQs with immediate practical application, and have developed according to the Grading of Recommendations Assessment, Development and Evaluation (GRADE) methodology $[13,14]$. Moreover, both guidelines address CQs relating to nonoperative treatment, as well as surgical treatment, for perforated PU (PPU); however, there are few reports that evaluate the characteristics and treatment outcomes of perforated cancer.

In this study, we consecutively investigated patients who were diagnosed with PUGT caused by PU or cancer. The first aim of this study was to evaluate the optimal treatment for PUGT patients; the second aim was to compare the postoperative complications of patients who have undergone surgery and to investigate the predictors of postoperative complications.

\section{Methods}

\section{Patients}

Sixty-two consecutive patients who were diagnosed with PUGUT, stomach, or duodenum between 2010 and 2019 were identified from the electronic database at Kinan Hospital. The patients with retroperitoneal air $(n=10)$, intestinal wall air $(n=1)$, and those receiving best supportive care for advanced gastric cancer $(n=1)$ were excluded; finally, the remaining 50 patients with intraperitoneal free air were analyzed. All patients received initial inpatient treatment that was categorized as either surgery $(n=43,86 \%)$ or nonsurgery $(n=7,14 \%)$ (supplement Fig. 1). All patients provided written informed consent for treatment and the use of clinical data.

The data on patient characteristics, treatment outcomes, and postoperative findings were reviewed from medical reports. Patient characteristics included age, sex, body mass index (BMI), pretreatment mean arterial pressure (MAP), pretreatment heart rate (HR), perforation-to-treatment duration, peritonitis, perforated organ, cause of perforation, location of ascites, location of intraperitoneal free air, and blood examinations. The location of ascites and intraperitoneal free air were detected by CT scan prior to the initial treatment. Surgical outcomes were evaluated by surgical procedures, the use of laparoscopy, perforation size, bleeding, peritoneal lavage, and operation time. The data of perforation size and 
peritoneal lavage were obtained from the surgical report. Renal failure was defined as less than $0.5 \mathrm{ml}$ $/ \mathrm{kg} / \mathrm{h}$ of urine output during surgery. The findings after surgical or non-surgical treatment were evaluated by the antibiotic administration period length, fasting period during treatment or after surgery, blood transfusion history, and the length of hospital stay. Postoperative complications were graded according to the Clavien-Dindo (CD) classification.

\section{Statistical analysis}

The results were expressed as median (range) for continuous variables and percentage for categorical variables. We retrospectively analyzed the associations between patient data and operative procedures or postoperative complications using $\chi^{2}$ tests and the Mann-Whitney $U$ test. A Cox proportional hazard regression analysis was used for univariate and multivariate analyses. All statistical tests were two-sided, and the threshold for statistical significance was $p=0.05$. Statistical analyses were performed with JMP® Pro 15.1.0 (SAS Institute Inc., Cary, NC, USA).

\section{Results}

\section{Comparison of patient characteristics between non-surgery and surgery groups}

We compared patient characteristics and treatment outcomes in the PUGT patients who were successfully cured by the initial treatment. The comparison between patients with non-surgery $(n=5)$ and those with surgery $(n=43)$ was then performed. Two non-surgery patients who were converted to surgery for worsening abdominal peritonitis and increased ascites the day after admission were excluded (Supplement Table 1). 
Table 1

Comparison of patient characteristic (Non-surgery vs Surgery)

\begin{tabular}{|c|c|c|c|c|c|}
\hline & Non- $S$ & gery & Surgery & & $\mathbf{P}$ \\
\hline & $N=5$ & & $N=43$ & & \\
\hline Age, years, median (range) & 48 & $(23-66)$ & 65 & $(30-92)$ & 0.029 \\
\hline Sex, n (\%) & & & & & 0.583 \\
\hline Male & 3 & $60 \%$ & 31 & $72 \%$ & \\
\hline Female & 2 & $40 \%$ & 12 & $28 \%$ & \\
\hline BMI, kg/m2, median (range) & 20.5 & $(17.7-24.5)$ & 21.2 & $(14.7-30.8)$ & 0.478 \\
\hline MAP (mmHg), median (range) & 98 & $(86-112)$ & 91.8 & $(60.7-115.0)$ & 0.558 \\
\hline HR (bpm), median (range) & 84 & $(80-99)$ & 88.5 & $(60-133)$ & 0.666 \\
\hline Perforation-to-treatment, n (\%) & & & & & 0.374 \\
\hline$<6$ & 3 & $60 \%$ & 24 & $55 \%$ & \\
\hline $6-12$ & 2 & $40 \%$ & 6 & $14 \%$ & \\
\hline $12-24$ & 0 & $0 \%$ & 8 & $19 \%$ & \\
\hline $24<$ & 0 & $0 \%$ & 5 & $12 \%$ & \\
\hline Peritonitis, n (\%) & & & & & 0.004 \\
\hline None & 3 & $60 \%$ & 0 & $0 \%$ & \\
\hline Localized & 2 & $40 \%$ & 19 & $44 \%$ & \\
\hline Generalized & 0 & $0 \%$ & 24 & $56 \%$ & \\
\hline Cause of perforation, n (\%) & & & & & 0.372 \\
\hline Ulcer & 5 & $100 \%$ & 37 & $86 \%$ & \\
\hline Cancer & 0 & $0 \%$ & 6 & $14 \%$ & \\
\hline Ascites*, n (\%) & & & & & \\
\hline None & 4 & $80 \%$ & 5 & $12 \%$ & 0.003 \\
\hline Perforated area & 1 & $20 \%$ & 38 & $88 \%$ & 0.003 \\
\hline Subphrenic area & 1 & $20 \%$ & 36 & $84 \%$ & 0.007 \\
\hline Douglas fossa & 0 & $0 \%$ & 21 & $49 \%$ & 0.059 \\
\hline Intraperitoneal free air, n (\%) & & & & & 0.569 \\
\hline
\end{tabular}




\begin{tabular}{|c|c|c|c|c|c|}
\hline & \multicolumn{2}{|c|}{ Non-Surgery } & \multicolumn{2}{|c|}{ Surgery } & \multirow[t]{2}{*}{$\mathbf{P}$} \\
\hline & \multicolumn{2}{|l|}{$N=5$} & \multicolumn{2}{|l|}{$N=43$} & \\
\hline Upper abdominal cavity & 5 & $100 \%$ & 33 & $77 \%$ & \\
\hline Entire abdominal cavity & 0 & $0 \%$ & 10 & $23 \%$ & \\
\hline \multicolumn{6}{|c|}{ Blood examination, median (range) } \\
\hline WBC $(/ \mu \mathrm{L})$ & 13200 & $(10300-20800)$ & 10000 & $(600-26600)$ & 0.085 \\
\hline Neutrophil $(/ \mu \mathrm{L})$ & 9780 & $(8990-17890)$ & 7800 & $(25800-350)$ & 0.107 \\
\hline $\operatorname{CRP}(\mathrm{mg} / \mathrm{dL})$ & 0.06 & $(0.01-6.12)$ & 1.72 & $(0.04-31.56)$ & 0.036 \\
\hline Hemoglobin (g/dL) & 14.6 & $(7.6-17.1)$ & 14.1 & $(6.5-18.1)$ & 0.893 \\
\hline Platelet $(/ \mu \mathrm{L})$ & 26.4 & $(18.4-44.4)$ & 24.4 & $(6.1-86.1)$ & 0.613 \\
\hline Creatinine (mg/dL) & 0.79 & $(0.5-1.6)$ & 0.87 & $(0.49-5.21)$ & 0.510 \\
\hline Total Bilirubin (mg/dL) & 1.1 & $(0.3-2.9)$ & 0.8 & $(0.3-3.9)$ & 0.270 \\
\hline AST (U/L) & 23 & $(15-155)$ & 20 & $(10-66)$ & 0.648 \\
\hline $\mathrm{ALT}(\mathrm{U} / \mathrm{L})$ & 13 & $(8-17)$ & 16 & $(5-69)$ & 0.427 \\
\hline PT-INR & 1.04 & $(0.95-1.19)$ & 1.09 & $(0.96-2.91)$ & 0.211 \\
\hline \multicolumn{6}{|c|}{$\begin{array}{l}\text { BMI, body mass index; MAP, mean arterial pressure; HR, heart rate; WBC, white blood cell, CRP; C- } \\
\text { reactive protein; PT-INR, prothrombin international normalized ratio }\end{array}$} \\
\hline${ }^{*}$ There are some duplicat & & & & & \\
\hline
\end{tabular}

No significant differences in sex, BMI, MAP, HR, perforation-to-treatment duration, cause of perforation, and location of intraperitoneal free air were found between the two groups. The non-surgery patients were significantly younger $(P=0.029)$ and had, also significantly, no or localized peritonitis $(P=0.004)$ compared to those who were surgically treated. No ascites was significantly detected by CT scan before treatment in four non-surgery patients $(P=0.003)$. The ascites in the perforated and subphrenic areas were detected in more than 80 percent of surgical patients and only one non-surgical patient $(P=0.003$ and $P=0.007$, respectively). Ascites was detected in the Douglas fossa in half of the surgical patients but not in the non-surgery patients $(P=0.059)$. $C$-reactive protein $(C R P)$ was significantly lower in the nonsurgery patients than the surgery patients $(P=0.036)$ (Table 1$)$.

\section{Comparison Of Treatment Outcomes Between Non-surgery And Surgery Groups}

Almost half of the patients in each group were treated with carbapenem as an initial antibiotic agent. The non-surgery patients tended to have a shorter period of antibiotic administration than those who underwent surgery $(P=0.080)$. No significant differences in the fasting period and blood transfusion were 
found between the two groups. The length of hospital stay was significantly shorter in non-surgery patients than in surgery patients $(P=0.016)$ (Table 2$)$.

Table 2

Comparison of posttreatment findings (Non-surgery vs Surery)

\begin{tabular}{|llllll|}
\hline & Non-Surgery & Surgery & P \\
\cline { 1 - 5 } & N=5 & N=43 & \\
\hline Antibiotic agent, $n(\%)$ & & & & & 0.235 \\
\hline$\beta$-lactam & 2 & $40 \%$ & 6 & $14 \%$ & \\
\hline 1 st Cephem & 0 & $0 \%$ & 4 & $9 \%$ & \\
\hline 2nd Cephem & 0 & $0 \%$ & 13 & $31 \%$ & \\
\hline Carbapenem & 3 & $60 \%$ & 19 & $44 \%$ & \\
\hline None & 0 & $0 \%$ & 1 & $2 \%$ & \\
\hline Antibiotic administration period (day), median (range) & 5 & $(3-6)$ & 6 & $(0-38)$ & 0.080 \\
\hline Fasting period (day), median (range) & 6 & $(5-9)$ & 6 & $(1-71)$ & 0.917 \\
\hline Blood Transfusion, $n$ (\%) & 0 & $0 \%$ & 15 & $35 \%$ & 0.167 \\
\hline Length of hospital stay (day), median (range) & 11 & $(8-15)$ & 19 & $(1-110)$ & 0.016 \\
\hline
\end{tabular}

Comparison Of Preoperative Patient Characteristics Classified By Postoperative Complications

We evaluated the postoperative complications of all patients who underwent surgery for PUGT according to the CD classification (Grade 0, $n=18(40 \%)$; Grade $\varangle, n=6(13 \%)$; Grade $\nabla, n=13(30 \%)$; Grade $\nabla, n=5$

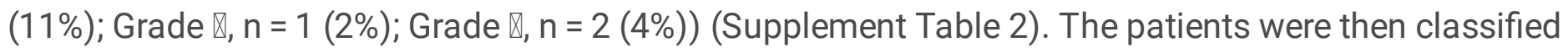

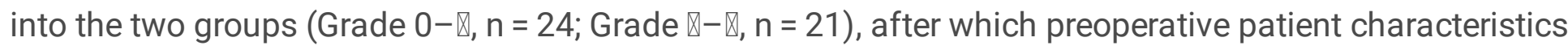
were compared (Table 3). No significant differences in sex, BMI, peritonitis, cause of perforation, and

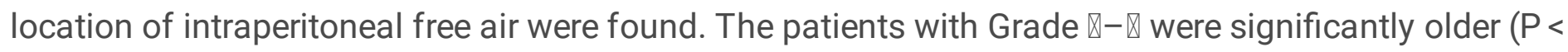
$0.001)$ and had higher HR $(P=0.001)$, poorer American Society of Anesthesiologists physical status classification system (ASA-PS) results $(P=0.031)$, and longer perforation-to-surgery $(P=0.032)$ than

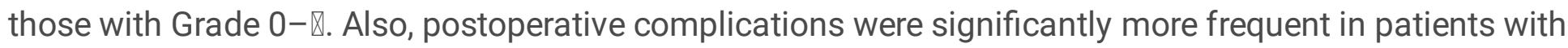
a perforated stomach $(P=0.006)$. Blood examinations showed that white blood cell $(W B C)$, neutrophil, and hemoglobin counts were significantly lower $(P=0.006, P=0.049$, and $P<0.001$, respectively), and CRP, prothrombin international normalized ratio (PT-INR), and lactate levels were significantly higher in Grade $\nabla-\otimes$ patients than Grade $0-\nabla$ patients $(P=0.001, P=0.004$, and $P=0.020$, respectively). 
Table 3

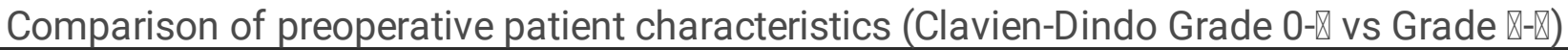

Grade 0-వ

$N=24$

58

Age (years), median (range)

Sex, $\mathrm{n}(\%)$

Male

Female

BMI ( $\mathrm{kg} / \mathrm{m} 2)$, median (range)

MAP (mmHg), median (range)

HR (bpm), median (range)

ASA-PS, n (\%)

$\begin{array}{ll}1 & 4 \\ 2 & 7 \\ 3 & 13 \\ 4 & 0\end{array}$

Perforation-to-surgery, n (\%)

$<6$

6-12

$12-24$

24<

Peritonitis, n (\%)

Localized

Generalized

Perforated organ, n (\%)

Stomach

Duodenum

Cause of perforation, $\mathrm{n}(\%)$

Ulcer

17

17

2

1

1

13

11
Grade $\mathbb{X}-\mathbb{X}$

$N=21$

$\mathbf{P}$

78

(51-92)

$<0.001$

0.176

$13 \quad 62 \%$

$8 \quad 38 \%$

$21.2 \quad(16.3-29.7)$

21.1

(14.7-30.8)

0.865

$94.3 \quad(69.3-120.7)$

89.7

(60.7-141.7)

0.056

$75 \quad(60-121)$

102

(60-133)

0.001

0.031

\begin{tabular}{|llll}
\hline 4 & $17 \%$ & 0 & $0 \%$ \\
\hline 7 & $29 \%$ & 3 & $14 \%$ \\
\hline 13 & $54 \%$ & 17 & $81 \%$ \\
\hline 0 & $0 \%$ & 1 & $5 \%$ \\
\hline
\end{tabular}

$71 \%$

8

$37 \%$

$4 \quad 17 \%$

2

$10 \%$

$8 \%$

6

$29 \%$

$4 \%$

5

$24 \%$

$54 \%$

8

13

$38 \%$

$46 \%$

$62 \%$

0.032 


\begin{tabular}{|c|c|c|c|c|c|}
\hline & \multicolumn{2}{|c|}{ Grade $0-\rrbracket$} & \multicolumn{2}{|c|}{ Grade $\rrbracket-\rrbracket$} & \multirow[t]{2}{*}{$\mathbf{P}$} \\
\hline & \multicolumn{2}{|l|}{$N=24$} & \multicolumn{2}{|l|}{$N=21$} & \\
\hline Cancer & 1 & $4 \%$ & 5 & $24 \%$ & \\
\hline \multicolumn{6}{|l|}{ Ascites, n (\%) } \\
\hline Perforated area & 19 & $79 \%$ & 20 & $95 \%$ & 0.193 \\
\hline Subphrenic area & 17 & $71 \%$ & 19 & $90 \%$ & 0.143 \\
\hline Douglas fossa & 8 & $33 \%$ & 13 & $62 \%$ & 0.076 \\
\hline Intraperitoneal free air, n (\%) & & & & & 0.476 \\
\hline Upper abdominal cavity & 20 & $83 \%$ & 15 & $71 \%$ & \\
\hline Whole abdominal cavity & 4 & $17 \%$ & 6 & $29 \%$ & \\
\hline \multicolumn{6}{|c|}{ Blood examination, median (range) } \\
\hline $\mathrm{WBC}(/ \mu \mathrm{L})$ & 12550 & $(4000-26600)$ & 7300 & $(600-17700)$ & 0.006 \\
\hline Neutrophil $(/ \mu \mathrm{L})$ & 10610 & $(1275-25800)$ & 5540 & $(350-15460)$ & 0.049 \\
\hline CRP (mg/dL) & 0.5 & $(0.04-30.89)$ & 10.83 & $(0.2-31.6)$ & 0.001 \\
\hline Hemoglobin (g/dL) & 15.55 & $(7.2-18.1)$ & 11.9 & $(6.5-17.1)$ & $<0.001$ \\
\hline Platelet $(/ \mu \mathrm{L})$ & 24.3 & $(15.5-36.8)$ & 27.1 & $(6.1-86.1)$ & 0.625 \\
\hline Creatinine (mg/dL) & 0.825 & $(0.57-1.41)$ & 1.12 & $(0.49-5.21)$ & 0.088 \\
\hline Total Bilirubin (mg/dL) & 0.8 & $(0.3-2)$ & 0.8 & $(0.3-3.9)$ & 0.723 \\
\hline AST (U/L) & 20 & $(14-69)$ & 20 & $(9-66)$ & 0.793 \\
\hline ALT (U/L) & 16 & $(5-69)$ & 14 & $(1-51)$ & 0.380 \\
\hline PT-INR & 1.07 & $(0.960-2.060)$ & 1.27 & $(1.00-2.91)$ & 0.004 \\
\hline Lactate (mg/dL) & 12 & $(7-32)$ & 19.5 & $(6-80)$ & 0.020 \\
\hline
\end{tabular}

Comparison of surgical outcomes and postoperative findings classified by postoperative complications

We compared surgical outcomes and postoperative findings in the two groups (Table 4). The surgical procedures were used omental plombage and patch $(n=17(71 \%), n=13(61 \%)$, respectively), simple closure with omental patch $(n=5(21 \%), n=6(29 \%)$, respectively), omental patch $(n=2(8 \%), n=1(5 \%)$, respectively), or gastrectomy $(n=0(0 \%), n=1(5 \%)$, respectively). No significant differences in surgical 
procedures, perforation size, use of laparoscopy, bleeding, and peritoneal lavage were found between the two groups. The patients with Grade $\otimes-\otimes$ had significantly longer operation times $(P=0.017)$. Acute renal failure was more frequently found in patients with Grade $\nabla-\otimes(P=0.002)$.

Table 4

Comparison of surgical outcomes and postoperative findings (Clavien-Dindo Grade $0-\nabla$ vs Grade $\rrbracket-\nabla$ )

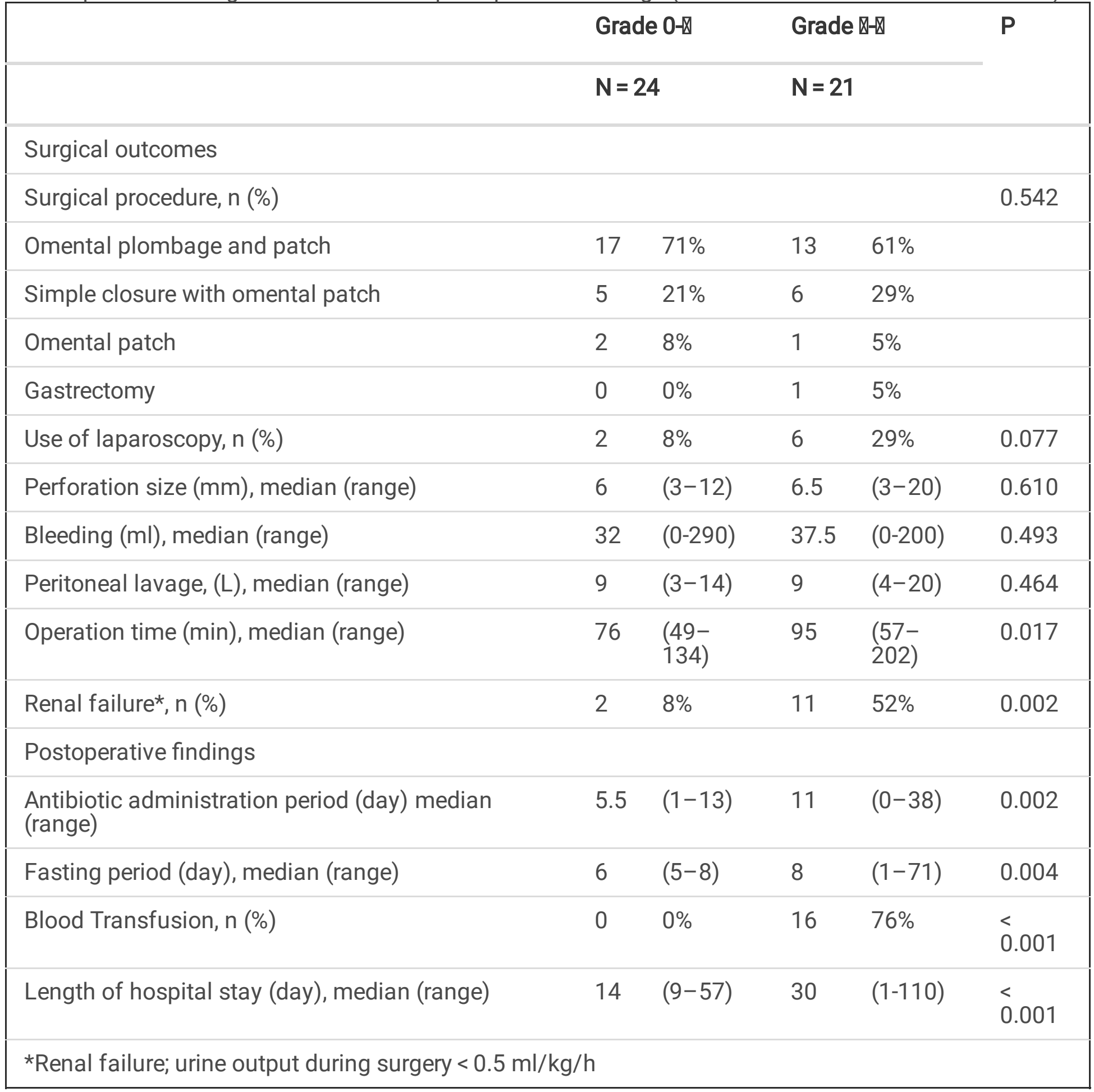

Postoperative findings showed a significantly more prolonged period of antibiotic administration, fasting, and hospital stay in patients with Grade $\nabla-\nabla$ than those with Grade $0-\otimes(P=0.002, P=0.004$, and $P<$ 0.001 , respectively). Significantly more patients with Grade $\nabla-\nabla$ received blood transfusions than those with Grade $0-\otimes(P<0.001)$. 


\section{Comparison Of Postoperative Blood Examinations Classified By Postoperative Complications}

Figure 1 shows the change in postoperative blood examinations (WBC, neutrophil, CRP, hemoglobin, and platelet counts) on postoperative days (POD) 1, 3, and 7 respectively. Minor changes were observed in WBC and neutrophil in patients with Grade $\nabla-\varangle$ after surgery. Neutrophil (POD7) and CRP (POD1, POD3, and POD7) were significantly higher in patients with Grade $\nabla-\nabla$ than in those with Grade $0-\nabla$ (neutrophil, $P=0.011 ; C R P, P=0.016, P=0.039$, and $P<0.001$, respectively).

\section{Evaluation of the risk factor of severe postoperative complications (CD Grade $₫ \leqq$ )}

The risk factor of postoperative complications (Grade $₫ \leqq$ ) was compared among the potential risk factors of preoperative patient characteristics (e.g. age, sex, ASA-PS, perforation-to-surgery, perforated organ, cause of perforation, preoperative HR, PT-INR, hemoglobin, CRP, and lactate). The univariate analyses showed that age ( $65 \leqq)$, higher ASA-PS ratings ( $3 \leqq)$, longer perforation-to-surgery (6 hours $\leqq$ ), perforated organ (stomach), high PT-INR (1.20 ), low hemoglobin count $(<12)$, high CRP levels $(10 \leqq)$,

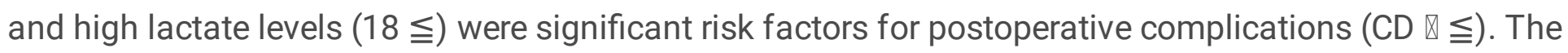
multivariable analyses identified lactate levels (18 $\leqq$ ) as an independent risk factor for postoperative complications ( $\mathrm{CD} \otimes \leqq$ ) (odds ratio 34.82, 95\% confidence interval [Cl] 1.03-1173.61, P=0.048) (Table 5). 
Table 5

Univariate and multivariate Cox model analysis for postoperative complications (Clavien-Dindo Grade

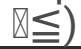

\begin{tabular}{|c|c|c|c|c|c|c|}
\hline & \multicolumn{3}{|c|}{ Univariate analysis } & \multicolumn{3}{|c|}{ Multivariate analysis } \\
\hline & $\begin{array}{l}\text { Odds } \\
\text { Ratio }\end{array}$ & {$[95 \% \mathrm{Cl}]$} & $P$ & $\begin{array}{l}\text { Odd } \\
\text { Ratio }\end{array}$ & {$[95 \% \mathrm{Cl}]$} & $P$ \\
\hline Age $(65 \leqq)$ & 10.32 & $\begin{array}{l}{[2.54-} \\
41.87]\end{array}$ & 0.001 & 3.90 & $\begin{array}{l}{[0.29-} \\
52.24]\end{array}$ & 0.304 \\
\hline Sex (Female) & 3.08 & $\begin{array}{l}{[0.77-} \\
12.34]\end{array}$ & 0.103 & & & \\
\hline 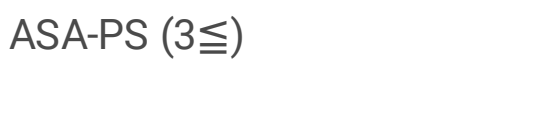 & 5.08 & $\begin{array}{l}{[1.18-} \\
21.91]\end{array}$ & 0.029 & 3.21 & $\begin{array}{l}{[0.15-} \\
70.36]\end{array}$ & 0.458 \\
\hline $\begin{array}{l}\text { Perforation-to-surgery (6 } \\
\text { hours } \leqq \text { ) }\end{array}$ & 3.95 & $\begin{array}{l}{[1.14-} \\
13.71]\end{array}$ & 0.031 & 5.35 & $\begin{array}{l}{[0.39-} \\
73.81]\end{array}$ & 0.210 \\
\hline Perforated organ (Stomach) & 6.67 & $\begin{array}{l}{[1.68-} \\
26.45]\end{array}$ & 0.007 & 14.59 & $\begin{array}{l}{[0.88-} \\
242.38]\end{array}$ & 0.062 \\
\hline $\begin{array}{l}\text { Cause of perforation } \\
\text { (Cancer) }\end{array}$ & 7.19 & {$[0.7767 .50]$} & 0.084 & & & \\
\hline Preoperative HR (94 bpm $\leqq$ ) & 6.67 & $\begin{array}{l}{[1.68-} \\
26.45]\end{array}$ & 0.007 & 3.32 & $\begin{array}{l}{[0.27-} \\
40.32]\end{array}$ & 0.347 \\
\hline 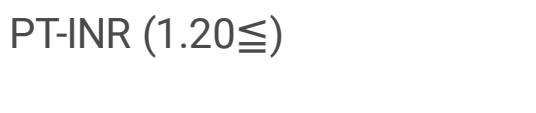 & 7.70 & $\begin{array}{l}{[1.75-} \\
33.90]\end{array}$ & 0.007 & 0.11 & $\begin{array}{l}{[0.004-} \\
3.11]\end{array}$ & 0.195 \\
\hline Hemoglobin $(12<)$ & 6.36 & $\begin{array}{l}{[1.44-} \\
28.02]\end{array}$ & 0.014 & 13.44 & $\begin{array}{l}{[0.65-} \\
276.83]\end{array}$ & 0.092 \\
\hline $\operatorname{CRP}(10 \leqq)$ & 12.10 & $\begin{array}{l}{[2.25-} \\
65.05]\end{array}$ & 0.004 & 45.37 & $\begin{array}{l}{[0.89-} \\
2310.88]\end{array}$ & 0.057 \\
\hline Lactate $(18 \leqq)$ & 7.70 & $\begin{array}{l}{[1.75-} \\
33.90]\end{array}$ & 0.007 & 34.82 & $\begin{array}{l}{[1.03-} \\
1173.61]\end{array}$ & 0.048 \\
\hline
\end{tabular}

\section{Discussion}

Most of the patients with suspected PPU received immediate surgery as an initial treatment [3, 9]. Recently, the possibility of non-operative management has been assessed in the WSES and JSGE guidelines for PPU [11, 12]. The WSES recommended against a routinely use of non-operative management, but non-operative management could be considered in extremely selected cases where perforation has sealed as confirmed on water-soluble contrast (weak recommendation based on lowquality evidence, 2C) [11]. The JSGE proposed that mild localized peritonitis could be an indication of nonoperative treatment for PPU. The criteria for conservative treatment include; onset within 24 hours, 
onset at hunger, stable condition without severe complication, symptoms of peritoneal irritation localized in the upper quadrant, and a small amount of ascites (Recommendation 2,100\% agreed, evidence level D) [12]. In our study, all patients, who were successfully cured by conservative treatment, were under 70 years of age, presented the perforation less than 12 hours earlier, were in a stable condition without severe complications, and had no or localized peritonitis and ascites located in the upper quadrant. Moreover, there were significant differences in these factors between surgery patients and non-surgery patients. On the other hand, nine patients who underwent surgery also matched the criteria of conservative treatment as laid out in the JSGE guidelines before surgery. Only one of them had postoperative complications with CD Grade $\otimes$ (aspiration pneumonia), but other patients were entirely cured without any postoperative complications. These results suggest that these patients might have had the potential to be cured without surgery, although careful observation should be needed during conservative treatment. The prospective study of conservative therapy for the PUGT based on the above guidelines should be pursued in future research.

The JSGE guidelines propose that patients with PPU should be surgically treated when clinical and imaging findings show no improvement after 24 hours of conservative treatment (Recommendation 2, $100 \%$ agreed, evidence level D) [12]. In our study, two patients were converted to surgery because clinical and imaging findings showed no improvement 24 hours after non-surgical treatment. One patient was 33 years of age and presented the perforation within 6 hours, localized peritonitis, and ascites only at the perforated area. He was discharged 11 days after surgery without any postoperative complications. Another patient showed no peritonitis and no ascites, but he was older (79 years of age), and had a longer perforation-to-treatment time (more than 24 hours). He stayed at the hospital 61 days after surgery with CD Grade $\otimes$. The WSES and JSGE guidelines both recommend performing surgery as soon as possible, especially in patients older than 70 years old and instances where hospital admission was delayed $[11,12,15,16]$. Buck et al. have shown that elderly patients may experience higher mortality. Every hour of delay from admission to surgery was associated with an adjusted $2.4 \%$ decreased probability of survival compared with the previous hour if non-operative management fails. Furthermore, caution is advised in patients $>70$ years of age [17]. Based on the guidelines and our own experiences, immediate surgery should be selected as an initial treatment for older patients and those whose treatment has been delayed.

In 2010, a systematic review including 50 studies was performed. Thirty-seven prognostic factors were assessed in a total of 29,782 patients who underwent surgery for PPU. The review provided strong evidence for an association of shock upon admission, preoperative metabolic acidosis, tachycardia, acute renal failure, low serum albumin level, high ASA score, and preoperative delay $>24 \mathrm{~h}$ with poor prognosis [18]. In our study, we compared the postoperative complications for surgical patients and examined the predictors of postoperative complications (Grade $₫ \leqq$ ). Older age, high ASA-PS, longer perforation-to-surgery duration, stomach perforation, tachycardia, abnormal coagulation, anemia, and elevated CRP and lactate levels were associated with postoperative complications. Moreover, the multivariable analyses also identified lactate $(18 \leqq)$, which is one of the septic shock criteria [19], as an independent risk factor for postoperative complications. These results support the appropriate targets for 
resuscitation, which include: MAP $\geq 65 \mathrm{mmHg}$, urine output $\geq 0.5 \mathrm{ml} / \mathrm{kg} / \mathrm{h}$, and lactate normalization, as suggested by the WSES guidelines [11]. In addition, the aforementioned systematic review reported that mortality was associated with older age, comorbidity, and use of NSAIDs or steroids [18]. In this study, the rate of mortality was $4 \%(\mathrm{~N}=2)$. One patient with abdominal symptoms approximately 2 days before surgery was elderly (79 years of age), and another patient with stomach perforation caused by advanced gastric cancer was in an unstable condition. They had not used NSAIDs or steroids, but both had preoperatively elevated lactate $(80 \mathrm{mg} / \mathrm{dL}, 19 \mathrm{mg} / \mathrm{dL}$, respectively), which was identified as an independent risk factor for postoperative complications in this study.

Spontaneous perforated gastric cancer (PGC) has an incidence ranging from $0.39-9.6 \%$ in gastric cancer patients as reported by different studies [4, 20-22]. It is difficult to diagnose PGC preoperatively because its preoperative symptoms are the same as those of a perforated gastric ulcer [4]. Therefore, the outcomes after emergency surgery in patients with free perforation depend on the stage and whether a curative resection can be performed [23]. Surgical management has usually been performed using onestage or two-stage surgery [24]. In this study, 4 of 6 patients with PGC could be diagnosed after the initial surgery; two patients received two-stage surgery (distal gastrectomy plus lymph node dissection), one patient received chemotherapy for unresectable, and one patient received best supportive care. The other patients were able to be diagnosed before the initial surgery because they received chemotherapy for gastric cancer. One patient underwent gastrectomy as a one-stage surgery. Another patient underwent simple closure with omental patch instead of gastrectomy because of multiple metastases. Five patients were dead within one year after surgery, while one gastrectomy patient (pStage $\mathbb{\otimes}$ ) has been alive with no recurrence for more than 5 years.

In terms of surgical procedures, some previous reports showed that the maneuver to cover an omental patch on the repaired PPU did not show additional advantage compared to simple closure alone [25-27]. The WSES suggested performing primary repair in patients with PPU smaller than $2 \mathrm{~cm}$, and no recommendation can be made whether the use of an omental patch can provide further protection of the repair (weak recommendation based on low-quality evidence, 2C) [11]. On the other hand, other research showed low postoperative leak rates with the omental patch technique, even in perforations of up to $2 \mathrm{~cm}$ in diameter [28], and suggested the adjunct of an omental patch to reduce the risk of the suture cutting through the edges of the ulcer [29]. The JSGE recommend peritoneal lavage in addition to the closure of the perforated hole with an omental patch as the surgical procedure for gastroduodenal PPU (Recommendation 1, 100\% agreed, evidence level A) [12]. In our institute, most of the patients with PUGT received omental plombage and patch $(n=30,68 \%)$ or simple closure plus omental patch $(n=11,25 \%)$. No simple closure alone has performed. The perforation size tended to be bigger in patients with omental plombage and patch than those with simple closure plus omental patch, but the selection of surgical procedures was not associated with postoperative complications.

The WSES recommended a laparoscopic approach in stable patients, but an open approach is recommended in the absence of appropriate laparoscopic skills and equipment (weak recommendation based on moderate-quality evidence, 2B) $[5,30]$. However, laparoscopic surgery for PPU is not included in 
the JSGE guidelines because the extent to which laparoscopic surgery is possible in the emergent setting depends upon the institution [12]. In our institute, laparoscopic surgery has mainly been used to confirm the location of the perforation, then laparoscopic surgery or open surgery was performed depending on the size of the perforation, the degree of peritonitis, and the skills of the surgeon with regards to approaching the perforation. Since 2018, the most frequently used method has been laparoscopy. Our study showed that the use of laparoscopy led to significantly less bleeding during surgery $(P=0.038)$, but it required a longer operation time $(P<0.001)$, a finding shared by other reports [30-32]. Patients who received laparoscopies tended to have more postoperative complications (Grade $0-\nabla, n=2$ (8\%); Grade $\nabla-$ $\nabla, n=6(29 \%), P=0.077)$; therefore, more careful and appropriate selection of surgical procedures is warranted.

The clinical presentation of gastroduodenal perforation is usually a sudden onset of abdominal pain. Localized or generalized peritonitis is typical of the perforated upper gastrointestinal tract, but may be present in only two-thirds of the patients [5-7]. Laboratory tests are also non-specific, although leukocytosis, metabolic acidosis, and elevated serum amylase are usually associated with perforation [33]. CT scan is increasingly taking the primary role in the diagnosis of perforation due to its greater sensitivity in detecting free air as well as its ability to characterize the site and size of perforation while excluding other possible causes $[6,10,34]$. Japan currently has over 100 CT scanners per million people, which is much higher than other countries [35]. In our institute, intraperitoneal free air could be identified in all patients by CT scan after their first outpatient visit. The appropriate early diagnosis by CT scan may have contributed to the reduction in mortality rates as compared to previous literature.

This study had some limitations. First, it was retrospective in design and the sample size was relatively small. Second, the survey period was relatively long, and there is a bias based on historical background, such as the induction of laparoscopic surgery and conservative treatment. Therefore, prospective, multicenter studies based on the aforementioned guidelines will be needed in the future.

\section{Conclusion}

Ten percent of patients with upper gastrointestinal perforation caused by PU were entirely cured by conservative treatment. The patients who received conservative treatment had relatively better treatment outcomes compared to those who underwent surgery, but more attention should be given to the indications of conservative treatment in the elderly and the delay of treatment, which was associated with poor short-term outcomes. In patients with surgery, several patient factors were associated with postoperative complications, and elevated lactate was identified as a risk factor for postoperative complications. The postoperative outcomes in patients with PGC depended on the stage and whether a curative resection could be performed. These results can be useful for the selection of treatment methods and the prediction of postoperative complications for PUGT patients.

\section{List Of Abbreviations}


PUGT: perforated upper gastrointestinal tract; PU: peptic ulcer; CT: computed tomography; WSES: World Society of Emergency Surgery; CQs: clinical questions; JSGE: Japanese Society of Gastroenterology; NSAIDs: non-nonsteroidal anti-inflammatory drugs; GRADE: Grading of Recommendations Assessment, Development and Evaluation; PPU: perforated peptic ulcer; BMI: body mass index; MAP: mean arterial pressure; HR: heart rate; CD: Clavien-Dindo; CRP: C-reactive protein; ASA-PS: American Society of Anesthesiologists physical status classification system; WBC: white blood cell; PT-INR: prothrombin international normalized ratio; POD: postoperative days; PGC: perforated gastric cancer

\section{Declarations}

\section{Ethics approval and consent to participate}

This retrospective study was performed in accordance with the guidelines of the Declaration of Helsinki. This study was approved by the Institutional Review Board of Kinan hospital (approval number 172). Written informed consent was provided by all patients who participated in this study

\section{Consent for publication}

Written informed consent for publication of their clinical details and clinical images was obtained from all patients.

\section{Availability of data and materials}

The datasets used and/or analyzed during the current study are available from the corresponding author on reasonable request.

\section{Competing interests}

The authors declare that they have no competing interests

\section{Funding}

No financial support was received in relation to this study.

\section{Authors' contributions}

Study concept and design: TS; data acquisition: TS; Analysis and interpretation of the data: TS, TM, and TH; drafting of the manuscript: TS, TM, SU, KS, TH, NH, and KY; statistical analysis: TS and TM; critical revision of the manuscript for intellectual content: TS and TM; study supervision: TS. All authors read and approved the submitted manuscript.

\section{Acknowledgments}

We would like to thank Editage (www.editage.com) for English language editing. 


\section{References}

1. Lanas A, Chan FKL. Peptic ulcer disease. Lancet. 2017;390(10094):613-24.

2. Søreide K, Thorsen K, Harrison EM, et al. Perforated peptic ulcer. Lancet. 2015;386(10000):1288-98.

3. Møller MH, Adamsen S, Thomsen RW, et al. Peptic Ulcer Perforation (PULP) trial group. Multicentre trial of a perioperative protocol to reduce mortality in patients with peptic ulcer perforation. Br J Surg. 2011;98(6):802-10.

4. Ignjatovic N, Stojanov D, Djordjevic M, et al. Perforation of gastric cancer - What should the surgeon do? Bosn J Basic Med Sci. 2016;16(3):222-6.

5. Søreide K, Thorsen K, Søreide JA. Strategies to improve the outcome of emergency surgery for perforated peptic ulcer. Br J Surg. 2014;101(1):e51-64.

6. Thorsen K, Glomsaker TB, von Meer A, et al. Trends in diagnosis and surgical management of patients with perforated peptic ulcer. J Gastrointest Surg. 2011;15(8):1329-35.

7. Suriya C, Kasatpibal N, Kunaviktikul W,et al. Diagnostic indicators for peptic ulcer perforation at a tertiary care hospital in Thailand. Clin Exp Gastroenterol. 2011;4:283-9.

8. Lim CH, Vani D, Shah SG, et al. The outcome of suspected upper gastrointestinal bleeding with 24hour access to upper gastrointestinal endoscopy: a prospective cohort study. Endoscopy. 2006;38(6):581-5.

9. Bertleff MJ, Lange JF. Perforated peptic ulcer disease: a review of history and treatment. Dig Surg. 2010;27(3):161-9.

10. Grassi R, Romano S, Pinto A, et al. Gastro-duodenal perforations: conventional plain film, US and CT findings in 166 consecutive patients. Eur J Radiol. 2004;50(1):30-6.

11. Tarasconi A, Coccolini F, Biffl WL, et al. Perforated and bleeding peptic ulcer: WSES guidelines. World J Emerg Surg. 2020;15:3.

12. Satoh K, Yoshino J, Akamatsu T, et al. Evidence-based clinical practice guidelines for peptic ulcer disease 2015. J Gastroenterol. 2016;51(3):177-94.

13. Guyatt $\mathrm{GH}$, Oxman AD, Kunz R, et al. Going from evidence to recommendations. BMJ. 2008;336(7652):1049-51.

14. Guyatt $\mathrm{GH}$, Oxman $\mathrm{AD}$, Vist $\mathrm{GE}$, et al. GRADE: an emerging consensus on rating quality of evidence and strength of recommendations. BMJ. 2008;336(7650):924-6.

15. Chung KT, Shelat VG. Perforated peptic ulcer - an update. World J Gastrointest Surg. 2017;9(1):1-12.

16. Crofts TJ, Park KG, Steele RJ, et al. A randomized trial of nonoperative treatment for perforated peptic ulcer. N Engl J Med. 1989;320(15):970-3.

17. Buck DL, Vester-Andersen M, Møller MH. Danish Clinical Register of Emergency Surgery. Surgical delay is a critical determinant of survival in perforated peptic ulcer. Br J Surg. 2013;100(8):1045-9.

18. Møller MH, Adamsen S, Thomsen RW, Møller AM. Preoperative prognostic factors for mortality in peptic ulcer perforation: a systematic review. Scand J Gastroenterol. 2010;45(7-8):785-805. 
19. Singer M, Deutschman CS, Seymour CW, et al. The Third International Consensus Definitions for Sepsis and Septic Shock (Sepsis-3). JAMA. 2016;315(8):801-10.

20. Roviello F, Rossi S, Marrelli D, et al. Perforated gastric carcinoma: a report of 10 cases and review of the literature. World J Surg Oncol. 2006;4:19.

21. Jwo SC, Chien RN, Chao TC, et al. Clinicopathological features, surgical management, and disease outcome of perforated gastric cancer. J Surg Oncol. 2005 Sep;15(4):219-25. 91(.

22. Kotan C, Sumer A, Baser M, et al. An analysis of 13 patients with perforated gastric carcinoma: $A$ surgeon's nightmare? World J Emerg Surg. 2008;3:17.

23. Tsujimoto $\mathrm{H}$, Hiraki S, Sakamoto $\mathrm{N}$, et al. Outcome after emergency surgery in patients with a free perforation caused by gastric cancer. Exp Ther Med. 2010;1(1):199-203.

24. Kandel BP, Singh Y, Singh KP, et al. Gastric cancer perforation: experience from a tertiary care hospital. JNMA J Nepal Med Assoc. 2013;52(191):489-93.

25. Vărcuş F, Beuran M, Lica I, et al. Laparoscopic Repair for Perforated Peptic Ulcer: A Retrospective Study. World J Surg. 2017;41(4):948-53.

26. Lo HC, Wu SC, Huang HC, Yeh CC, Huang JC, Hsieh CH. Laparoscopic simple closure alone is adequate for low risk patients with perforated peptic ulcer. World J Surg. 2011;35(8):1873-8.

27. Ates M, Sevil S, Bakircioglu E, Colak C. Laparoscopic repair of peptic ulcer perforation without omental patch versus conventional open repair. J Laparoendosc Adv Surg Tech A. 2007;17(5):6159.

28. Gupta S, Kaushik R, Sharma R, Attri A. The management of large perforations of duodenal ulcers. BMC Surg. 2005;5:15.

29. Siow SL, Mahendran HA. Laparoscopic repair of perforated peptic ulcers: the sutured omental patch and focused sequential lavage technique. Surg Laparosc Endosc Percutan Tech. 2014;24(2):134-9.

30. Cirocchi R, Soreide K, Di Saverio S, et al. Meta-analysis of perioperative outcomes of acute laparoscopic versus open repair of perforated gastroduodenal ulcers. J Trauma Acute Care Surg. 2018;85(2):417-25.

31. Bergström M, Arroyo Vázquez JA, Park PO. Self-expandable metal stents as a new treatment option for perforated duodenal ulcer. Endoscopy. 2013;45(3):222-5.

32. Alvarado-Aparicio HA, Moreno-Portillo M. Multimedia article: management of duodenal ulcer perforation with combined laparoscopic and endoscopic methods. Surg Endosc. 2004;18(9):1394.

33. Malhotra AK, Fabian TC, Katsis SB, et al. Blunt bowel and mesenteric injuries: the role of screening computed tomography. J Trauma. 2000;48(6):991-1000.

34. Yeung KW, Chang MS, Hsiao CP, et al. CT evaluation of gastrointestinal tract perforation. Clin Imaging. 2004;28(5):329-33.

35. He L, Yu H, Shi L, et al. Equity assessment of the distribution of CT and MRI scanners in China: a panel data analysis. Int J Equity Health. 2018;17(1):157. 
Figures
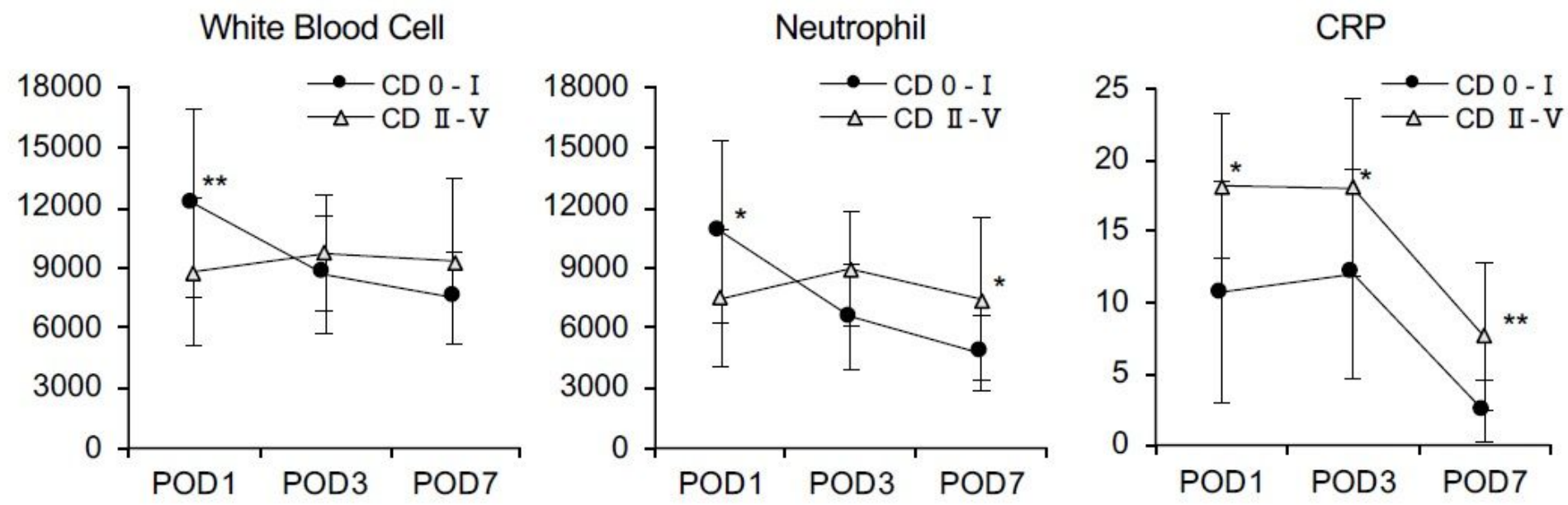

Hemoglobin
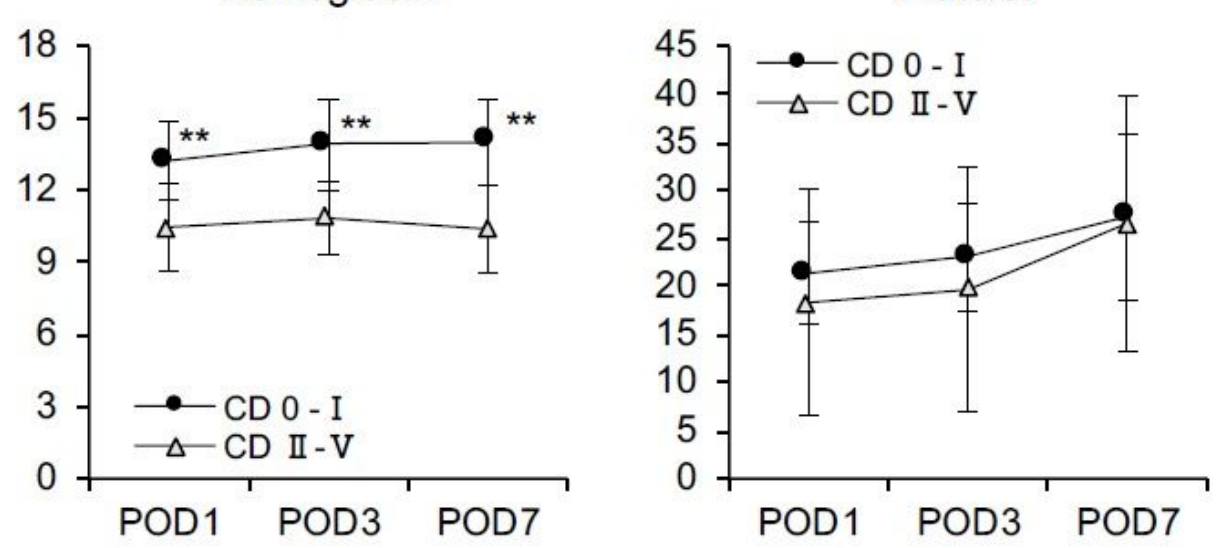

\section{Figure 1}

Comparison of postoperative examination between patients with Clavien-Dindo (CD) Grade 0 - $\nabla$ and Grade $\nabla-\nabla$. (a) white blood cell (WBC), (b) neutrophil, (c) C-reactive protein (CRP), (d)hemoglobin, (e) platelet. ${ }^{*} \mathrm{P}<0.050, * * \mathrm{P}<0.001$

\section{Supplementary Files}

This is a list of supplementary files associated with this preprint. Click to download.

- SupplementalTable1.docx

- SupplementalTable2.docx

- supplementalFigure1.pdf 